\title{
The Rock Pressure Detection System Based on the Vibrating Wire Sensor
}

\author{
Guan Gong1,a, Bin Wang 2,b \\ 1Department of Electric Engineering, Anhui University of Science and \\ Technology, Huainan, China \\ 2Department of Electric Engineering, Anhui University of Science and \\ Technology, Huainan, China \\ aemail:784758843@qq.com, bemail:perfect_co@qq.com
}

\begin{abstract}
This paper introduces a rock pressure detection system based on one coil vibrating wire sensor, with STM32F103C8T6 as main control chip, using feedback low voltage sweep excitation method, making the vibrating string resonance state in a short time.The output signals of sensor after dealing with the two level signal amplification circuit, band-pass filter circuit and the hysteresis comparator circuit, are collected by input capture unit of the main control chip, and the collected signals are transmitted to inoue PC over a long distance through power carrier communication module after temperature compensation at the same time, then achieving the mine pressure real-time online detection .

Keywords: vibrating string type pressure sensor; feedback type low voltage sweep

\section{Introduction}

With the rapid development of China's society and economy, coal industry gradually to high-tech, low resources consumption, little environmental pollution and direction, and further achieve more productive and efficient mining. But with the deepening and gradually increase the intensity of mining production, will always occur, such as roadway deformation, rock pressure shocks, roof off and other accidents, to mine brought serious economic losses, but also endanger the lives and safety of mine workers. So, in the mining process real-time data acquisition mine pressure, abnormal situation in a timely manner in the event of an alarm, can effectively prevent the occurrence of mine. The traditional method of mining pressure monitoring has the following disadvantages in harsh environments, multiple confounders mine: the accuracy of the measurement data is low, high complexity sensor installed, the high cost of the entire system. The design uses a vibrating wire pressure sensor, the output signal is a signal having a frequency distortion, high accuracy and strong anti-interference ability of the advantages that can achieve long-range detection in the coal mine.
\end{abstract}

\section{Basic principles}


The main components of vibration string pressure sensor is mainly made of steel strings, the main material just strings of tungsten steel,spring steel and Markov resilient stainless steel. Enough to drive the coil when the coil plus pulse frequency vibration signal, the core will produce pulsating magnetic field to drive the vibration of iron in the magnetic field. while the vibrating strings vibrate, vibrating wire in the start-up will produce an irregular shock attenuation signal that the magnetic lines cutting the core. while the attenuation through the coil output terminal of the sensor sine wave.

Single coil vibrating wire pressure sensor that uses an internal sensor excitation and sensing coils to achieve the vibrating string signal acquisition, work part due to vibration and frequency acquisition part time-sharing processing. So it is also called single coil intermittent vibration sensor,the biggest advantage is that the structure is simple,the installation is also more convenient.

\section{Pressure and Compensation Formula}

The working principle of vibrating string type pressure sensor is through rather than on the natural frequency of the steel string plus pressure formula to measure the size of the pressure, and its natural frequency is calculated as follows:

$$
\mathrm{f}=\frac{1}{2 L} \sqrt{\frac{T}{\rho}}
$$

\section{(1)}

The $f$ is the inherent frequency of vibrating string. $L$ is the length of the vibrating string. $T$ for the stress on the vibrating string. $P$ is the density of vibrating string.

Used in the sensor, temperature changes obviously will directly influence the accuracy of measurement data. The design of compensation by software, the compensated pressure calculation formula for:

$$
F=K\left(f_{0}^{2}-f_{1}^{2}\right)+\alpha
$$

$\mathrm{F}$ in the type was the string tension. $\mathrm{K}$ is the sensitivity coefficient of sensor. Natural frequency of the $\mathrm{f} 1$ for stress after just string.Standard natural frequency f0 is just strings. $\alpha$ For the correction factor.Correction factor is calculated as follows:

$$
\alpha=G\left(T_{1}^{2}-T_{2}^{2}\right)
$$

T1 is the vibrating wire current temperature of their environment. T2 standard for vibrating wire temperature. $G$ is vibrating wire temperature coefficient.

\section{Overall System Architecture}

Vibrating wire sensor mine pressure detection system mainly consists of the minimum system of the controller,vibrating wire sensor excitation circuit,pick up 
the monitoring systen of vibration circuit, signal isolation circuit, power line carriercommunication circuit, upper machine based on.The pick-up circuit includes a two stage signal amplifier circuit ,band-pass filter,schmidt shaping circuit.

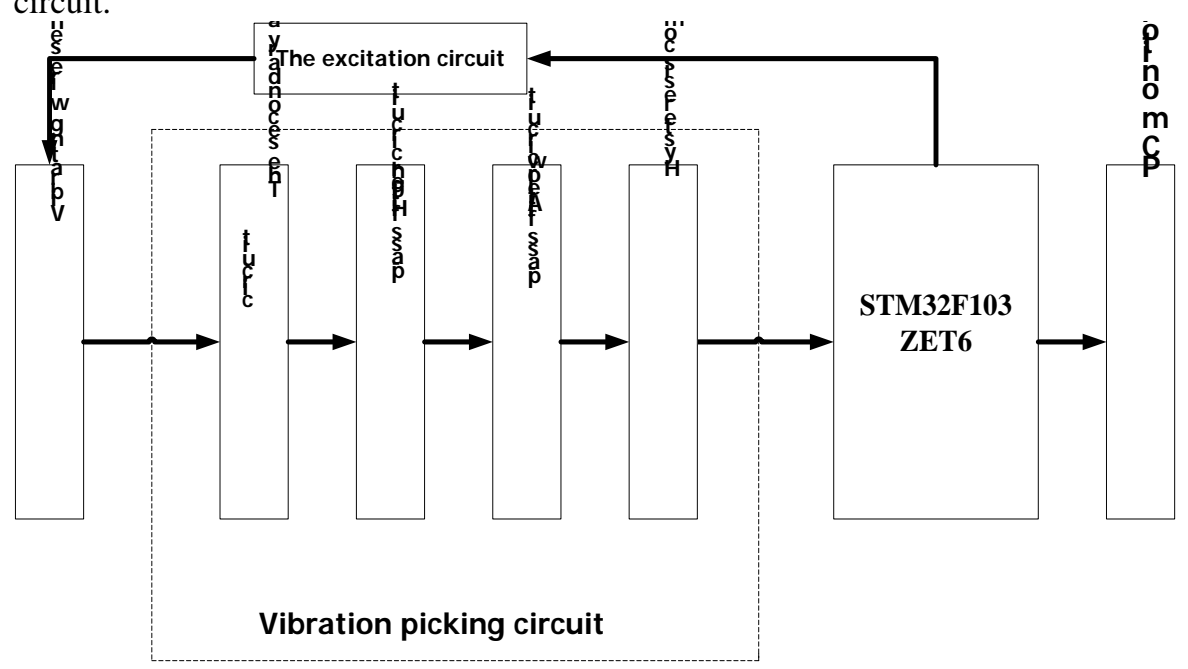

Figure 1. The system block diagram

\section{Gardware Design Part}

At present, vibrating wire sensor excitation method mianly has the low vibration frequency and vibration of two kinds of high voltage, low sweep approach is applied vibrating wire sensors low square wave excitation signal, select the square wave oscillator frequency segment depends natural frequency of the wire sensor. When the frequency of the excitation signal is close to the natural frequency of the sensor just strings, steel strings can quickly reach resonance and greater output frequency signal induced electromotive force, exciting way to generate high-pressure high-voltage fly back pulse signal booster circuit exciting steel strings, in the role of high-voltage pulse signal output sinusoidal voltage signal attenuation.

The system uses low-voltage feedback sweep exciting way, the first of vibrating wire sensors pre-excitation.Through to a single $5 \mathrm{~V}$ voltage excitation voltage is disconnected, just string free vibration and the output voltage signal is weak. Capture its output the voltage and frequency signals, and many times the pre-excitation mean value. If the average value at nominal frequency range of vibrating wire sensors, put it as the output frequency signal of complex mode, so just string quickly reach the resonance condition and halt vibration, resonance frequency samping just string output. Otherwise use sweep pre excitation mode, multiple sweep frequency and sampling averaging, as feedback frequency signal complex vibration. The software flow chart of excitation strategies as shown below: 


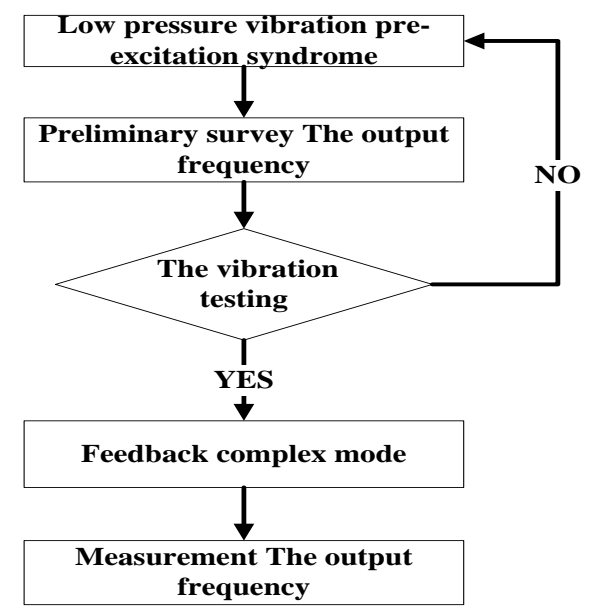

Figure 2. The strategy of software flow chart

Excitation circuit adopts the triode drive ability, increase the excitation signal and the sine fly-wheel diode in parallel on both ends of the sensor, prevent the disconnect excitation signal when the sensor inside the coil is damaged. The frequency of the vibrating wire sensor output signal attenuation of the sine voltage signal for mv level, this design uses the two-stage amplifier circuit of signal amplification, including operational amplifier should meet high input impedance, low drift, low output impedance, signal after dealing with the preamplifier should be carried out after a band pass filter signal filtering, to simplify the design, the band-pass filter consists of two parts, first part for the second-order active low-pass filter, the cut-off frequency of $4500 \mathrm{hz}$, after level for second-order active high-pass filter, the cut-off frequency of $400 \mathrm{hz}$. After filtering signal also need after schmidt shaping circuit and voltage clamping circuit, output pulse signal which is available for micro controller input capture circuit to count. Vibrating wire sensor excitation circuit and vibration picking circuit as shown in figure 3 and figure 4 :

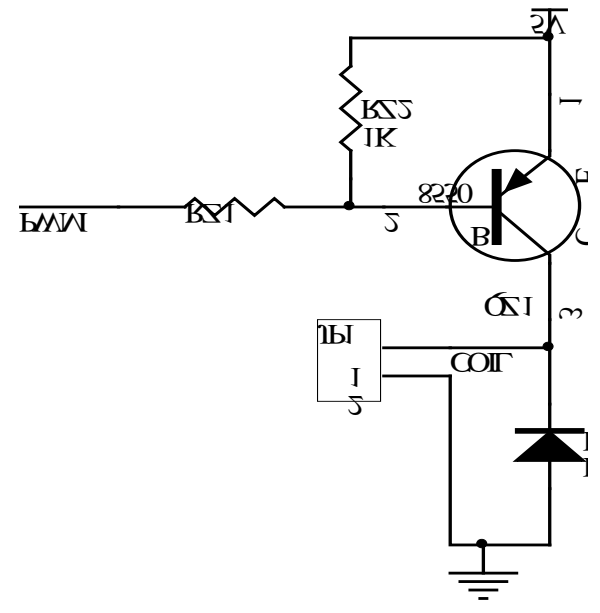


Figure 3.Vibrating wire sensor excitation circuit

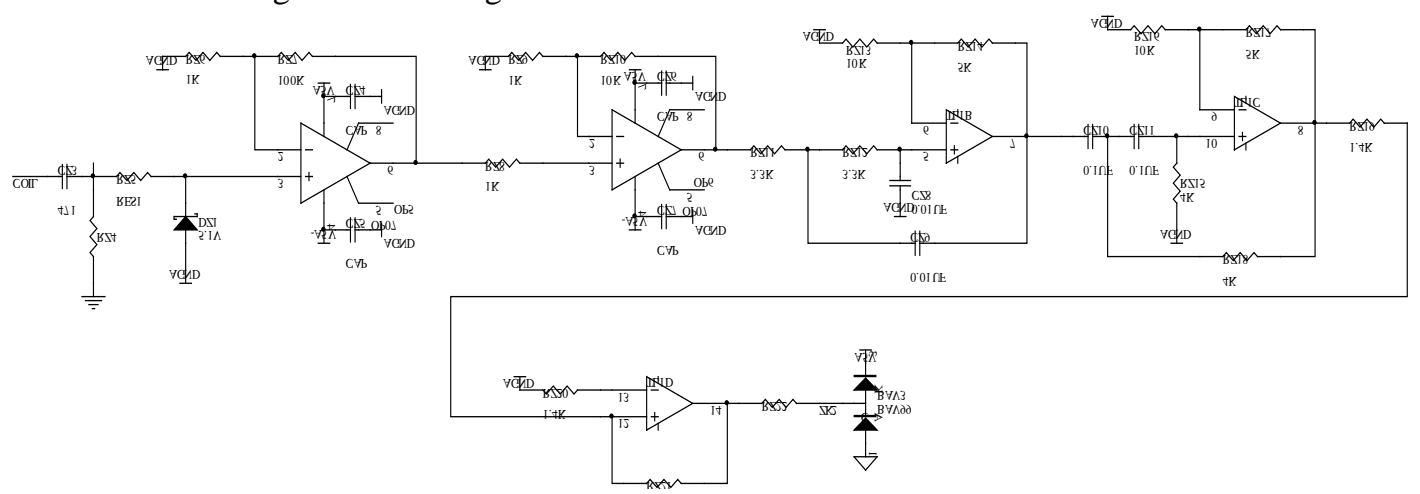

Figure 4. Vibrating wire sensor circuit vibration picking

\section{Experimental verification}

Continuous sampling of sensor samples for many times, the measured value distribution as shown:

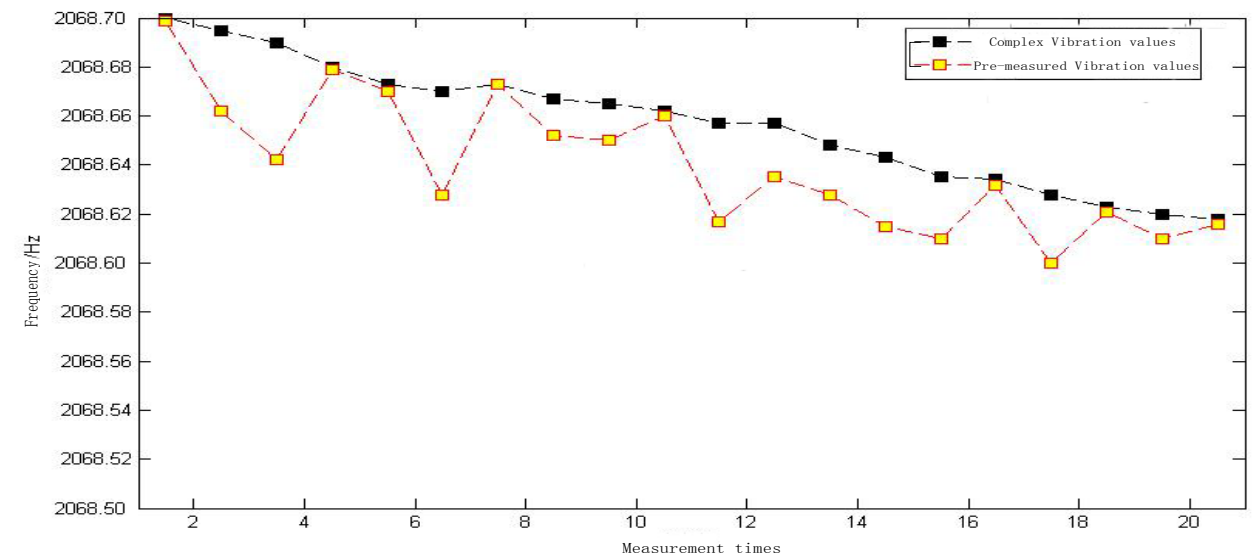

Figure 5.Measured value analysis

Obtained from the measurement curves, feedback vibration measure and low pre excitation deviation value is very close, proved that the low pressure pre excitation mode can obtain the effective signal, but the feedback excitation way measured signal on the repeat ability and stability is better. Frequency decreases slowly phenomena appear in the figure, is the result of long time working with a steel string, elevated temperature induced.

\section{Conclusion}

A feedback type low voltage sweep excitation improves the stability of the output signal of vibrating wire sensors, shortening the period of detection 
data, and through the software multiple sampling signal filtering, to improve the accuracy of data. This design has successfully built a pressure detection system of single coil vibrating wire sensor based on dynamic change of mine, can display real-time mine pressure, provides a powerful data for predicting the occurrence of accidents in mines.

\section{Acknowledgements}

The college students' Innovative and Entrepreneurship foundation of Anhui Province(Project No.201310361224)

\section{References}

[1] HE Hu, Optimization of Vibrating Wire Sensor Excitation Strategy [J], CHINESE JOURANAL OF SENSORS AND ACTUATORS, 2010.

[2] ZHAI Wen Sheng, Vibrating Wire Sensor Based on Frequency Measurement system Design [J], Journal of Henan Normal University,2011.

[3] Xu Chengjun, Research on Feasibility of Vibrating Wire Sensor in Dynamic Testing[J], Journal of Wuhan University of Technology,2012.

[4] Miao Xiao zhong, Development of Signal Acquisition Card for Vibrating-wire Transducers[J], Instrument Technique and Sensor, 2010.

[5]YING Xiong Chun,Design of the Diameter String Type Pressure Sensor[J], Instrument Technique and Sensor,2003. 